\title{
Dieta y comportamiento alimentario de un grupo de mico maicero Cebus apella de acuerdo a la variación en la oferta de frutos y artrópodos, en la Amazonía colombiana
}

\author{
Carolina GÓMEZ-POSADA ${ }^{1}$
}

\section{RESUMEN}

Se analizó la relación entre la abundancia de artrópodos y frutos maduros, con el comportamiento alimentario y dieta de un grupo de Cebus apella en la Amazonía colombiana durante 1999. La oferta de frutos se midió por transectos fenológicos y la abundancia de artrópodos por capturas manuales. La oferta de ambos recursos varió a lo largo del año, lo que permitió establecer tres períodos de disponibilidad combinada: abundancia, oferta intermedia y escasez. Se completaron 708 horas de seguimiento al grupo y con el método de barrido lento se determinó una dieta omnívora, siendo principalmente insectívora - frugívora (48.8\% - 42.9\%). Los maiceros consumieron 96 especies de plantas y la diversidad mensual de frutos en la dieta, no se correlacionó con la oferta de los mismos. En los meses de abundancia de recursos, tanto frutos como artrópodos fueron consumidos en altas cantidades, y los frutos presentaron una distribución agrupada. Durante los períodos de escasez de recursos, la dieta incluyó pocas especies de frutos, considerados clave, de difícil manipulación, como Cariniana micrantha (Lecythidaceae), y fue complementada con partes vegetales. A diferencia de otros estudios, durante la escasez de frutos, no se presentó el mayor consumo de artrópodos, puesto que éstos igualmente escaseaban. Los maiceros mostraron una dieta generalista - oportunista y utilizaron los recursos en la proporción en la que estuvieron disponibles. Así por ejemplo, los valores de selectividad para frutos fueron muy bajos, y las tasas de éxito de captura de artrópodos se correlacionaron con la disponibilidad de los mismos.

PALABRAS CLAVE: capuchinos, ecología alimentaria, bosques húmedos de tierras bajas, abundancia de frutos y artrópodos

\section{Diet and feeding behavior of a group of brown capuchin monkeys Cebus apella according to fruits and arthropods availability, in the Colombian Amazon}

\begin{abstract}
The relation between the abundance of fruits and arthropods, and the diet and feeding behavior of a group of Cebus apella was studied in the Colombian Amazon during 1999. Fruit availability was measured using phenological transects, and the abundance of arthropods by using manual captures. The abundance of both resources varied throughout the year, which allowed establishing three periods of availability: abundant, intermediate, and scarce availability. 708 hours of monkey observations were completed, and using slow scans, it was determined that they had an omnivorous diet, being principally insectivorous frugivorous (48.8\% - 42.9\%). Capuchins consumed 96 plant species, and the monthly diversity of fruits in the diet was not correlated with the fruit availability. During months of resource abundance, both arthropods and fruits were eaten in high quantities, and fruits showed clumped distribution. During fruit and arthropod shortage periods, the diet included a few key fruit species of hard manipulation, such as Cariniana micrantha (Lecythidaceae), and was complemented by vegetable parts. Unlike other studies, during the fruit shortage, the capuchins did not present the higher consumption of arthropods, since these items were also scarce. Capuchins showed an opportunistic diet, and the resources were used in the same proportion in which they were available. For example, the values of fruit selectivity were very low, and the arthropods capture rates were correlated with their availability.
\end{abstract}

KEYWORDS: tufted capuchins, feeding ecology, humid lowland forest, fruits and arthropods availability.

${ }_{1}$ Departamento de Biología, Universidad del Valle, Colombia. Dirección actual: Department of Biology, University of Washington. cgomez@u.washington.edu. 


\section{INTRODUCCIÓN}

Cebus apella (mono maicero, familia Cebidae) es un primate de tamańo mediano $(-3.7 \mathrm{~kg})$, con un amplia distribución geográfica en Sur América. Su dieta omnívora consta principalmente de insectos y frutos, pero también incluye partes vegetales, nueces y pequeños vertebrados, entre otros (Fragaszi et al. 2004). La dieta de los maiceros varía ampliamente dependiendo de la oferta de frutos en el bosque. Así, cuando la disponibilidad de frutos disminuye, esta especie puede concentrar su dieta en uno o pocos recursos clave, por ejemplo nueces (Terborgh 1983; Spironello 1991; Peres 1994) y semillas (Peres 1991); o puede ampliar la dieta, incrementando el consumo de insectos, flores, y partes vegetales (Brown y Zunino 1990; Galetti y Pedroni 1994; Zhang 1995).

No sólo la oferta de frutos cambia estacionalmente para los primates. Las poblaciones de artrópodos sufren cambios drásticos en su abundancia y composición en el bosque tropical, generalmente asociados al patrón de lluvias (Janzen 1973; Boinski y Fowler 1989). Estos factores pueden provocar cambios en las tácticas por parte de los consumidores, dentro de sus capacidades de adaptación. Por ejemplo, para Cebus nigritus en Brasil se han detectado cambios en las tácticas de forrajeo de insectos, relacionados con cambios en el tipo de presa capturada (Rímoli et al. 2008). A pesar de que los artrópodos ocupan usualmente cerca del $50 \%$ de la dieta de $C$. apella (Terborgh 1983; Stevenson et al. 2000; Gómez-Posada et al. 2003a), el efecto de su abundancia sobre la ecología de la especie no ha sido estudiado. Sólo existe información acerca de tipos de forrajeo y la relación entre las tasas de éxito de captura de artrópodos de acuerdo con la precipitación (Terborgh 1983). Sin embargo, en la gran mayoría de trabajos, no se tiene en cuenta la variación temporal en la disponibilidad de artrópodos. Es muy probable que para este primate, el más "insectívoro" dentro del género Cebus (Defler 2004), la abundancia de artrópodos afecte sus respuestas ecológicas. Es posible que las diferentes estrategias encontradas para esta especie en diferentes estudios, se deban no sólo a la abundancia y distribución de frutos, sino también a la disponibilidad de invertebrados.

Este estudio busca determinar cómo varía el comportamiento alimentario de $C$. apella de acuerdo a los cambios en la disponibilidad de sus dos recursos principales: frutos y artrópodos. Se trabaja bajo la hipótesis de que la abundancia de ambos recursos, afectan la ecología alimentaria de esta especie. C. apella, especie omnívora, ofrece una interesante oportunidad para comparar cómo estas dos fuentes de alimento afectan sus respuestas ecológicas y por lo tanto, su plasticidad y adaptabilidad a las características del medio.

La taxonomía de los maiceros cachones no ha sido resuelta y algunos autores reconocen a la especie presente en la Amazonía colombiana como C. macrocephalus (Rylands y Mittermier 2009). Este trabajo sigue la clasificación de Defler (2004) y Fragrazsy et al. (2004), quienes reconocen dicha especie como Cebus apella.

\section{MATERIALES Y MÉTODOS}

\section{Área de estudio}

El estudio se llevó a cabo en un bosque continuo, húmedo tropical de tierras bajas en la Estación Biológica Mosiro Itajura Caparú (EBMIC), ubicada en el Parque Nacional Natural Yaigojé-Apaporis (1060603 ha) en la Amazonía colombiana (105.55' S, 69³0.8' W), a 200 m de altitud, al sur oriente del Departamento del Vaupés (Figura 1). El promedio anual de temperatura es de $25.1^{\circ} \mathrm{C}$, precipitación anual de $3836 \mathrm{~mm}$, con un régimen bimodal estacional, sin una clásica estación seca (Defler y Defler 1996). Durante 1999 la precipitación fue de 4089 mm; la época lluviosa comprendió de abril a junio y un pico menor en enero. Los períodos de baja precipitación abarcaron de febrero a marzo y de julio a diciembre. El bosque presenta un dosel alto, entre 25 a $30 \mathrm{~m}$ de altura, con emergentes hasta de $45 \mathrm{~m}$. En la zona se diferencian seis tipos de bosques: colina, terraza, igapó, trancisión, bajo y caatinga. Las familias de plantas dominantes tanto en número como en especie son Leguminosae, Lecythidaceae, Moraceae, Myristicaceae y Palmae (para detalle de las especies vegetales típicas de cada tipo de bosque, ver Defler y Defler 1996). En la EBMIC conviven simpátricamente ocho especies de primates: Cebus apella, C. albifrons, Saimiri sciureus, Callicebus torquatus, Aotus vociferans, Alouatta seniculus, Lagothrix lagothricha y Cacajao melanocephalus (Defler y Defler 1996; Defler 2004).

\section{Oferta de recursos}

Para estimar la abundancia de artrópodos, se establecieron mensualmente 15 parcelas de $5 \mathrm{~m}^{2}$ x $3 \mathrm{~m}$ de alto (Boinski

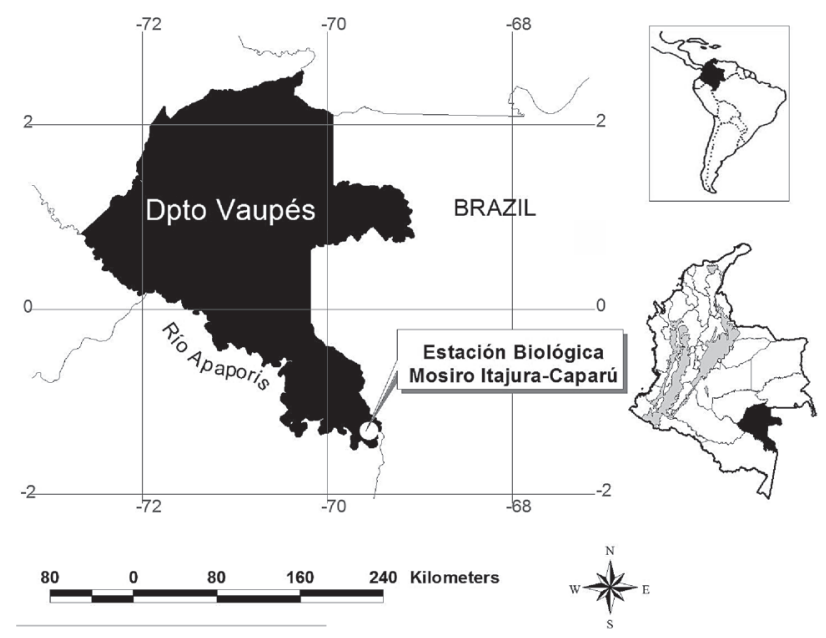

Figura 1 - Localización del área de estudio 
1987; Boinski y Fowler 1989) distribuidos al azar en el área de actividad del grupo en estudio. En cada parcela fueron colectados artrópodos durante una hora de forma manual (Boinski y Fowler 1989): buscando y revisando dentro de sustratos específicos, como por ejemplo dentro de troncos muertos, debajo de la corteza de árboles, en hojarasca suspendida, haz y envés de hojas vivas, superficie de tallos y dentro de flores. El método fue practicado durante un mes, previo a iniciar la toma de datos. Los artrópodos fueron capturados con la mano, con la ayuda de un frasco colector letal de boca ancha. Los muestreos fueron distribuidos equitativamente cada mes, entre las 9:00 y las 15:00 horas. No se realizaron muestreos bajo lluvia. Se contó el número de individuos colectados en cada parcela y se identificaron a nivel de orden. La abundancia mensual se tomó como el promedio de los individuos colectados en las 15 parcelas. La variación en el número de artrópodos colectados cada mes fue analizada por medio de una ANOVA de una vía.

En este estudio, los muestreos manuales fueron complementados con muestreos con red aérea. Sin embargo, los principales grupos de artrópodos colectados con la red no fueron consumidos por los maiceros (ver Gómez-Posada 2003b). Por tanto, se trabajó solamente con los resultados de los muestreos manuales.

La disponibilidad mensual de frutos maduros fue estimada por medio de 5 transectos fenológicos $(400 \times 5 \mathrm{~m})$ establecidos en el área de actividad del grupo. En cada transecto fue contado el número de árboles con frutos maduros mayores a $3 \mathrm{~m}$ de altura y el número de frutos maduros por árbol. La abundancia mensual se tomó como el promedio de los árboles con fruto maduro en los cinco transectos. Para analizar si el número total de árboles con fruto varió entre los meses del año, fue realizada una prueba de Kruskal-Wallis.

Para los artrópodos, fueron considerados los meses de escasez o de abundancia, como aquellos en los cuáles el número de artrópodos colectados fue significativamente menor o mayor al promedio mensual (calculado como el promedio entre todos los meses de muestreo). El mismo procedimiento se realizó para establecer los meses de escasez y abundancia de frutos maduros. Los métodos usados para estimar la abundancia de frutos y artrópodos no son estrictamente comparables. Sin embargo, para obtener un patrón de disponibilidad combinada de los dos recursos, se tuvo en cuenta los meses en que coincidió la escasez o abundancia de éstos (Raemaekers 1980). Así, cuando ambos recursos fueron abundantes, se consideró una época de abundancia; de escasez en aquellos meses en que ambos recursos escasearon, y de disponibilidad intermedia, cuando uno de los dos recursos presentó una baja oferta. Al sobreponer las gráficas de abundancia de artrópodos y frutos, con los puntos inferior y superior a la misma escala sobre el eje $Y$, se puede obtener una gráfica de la abundancia de los dos recursos de forma combinada (Raemaekers 1980).

\section{Dieta y comportamiento alimentario}

Durante el ańo de estudio, fueron registrados 5 grupos de maiceros, los cuales tienen su área de actividad o parte de éste dentro del sistema de senderos de la EBMIC. La composición de los grupos varió de 4 a 10 individuos. De éstos, fue seleccionado, habituado y seguido un grupo de 5 maiceros (1 macho adulto, 2 hembras adultas, un subadulto y una hembra juvenil) durante 5 días por mes, desde el amanecer cuando el grupo abandonó el dormitorio, hasta el atardecer, cuando llegaba a otro dormitorio, entre enero y diciembre de 1999. El área de actividad de este grupo fue de 367 ha, con recorridos diarios de 3202 \pm 576 m (Gómez-Posada 2003b). Fueron completadas 708 horas de seguimiento efectivo; durante el mes de marzo no se pudo contactar el grupo focal. Durante los días de fuertes lluvias, las observaciones fueron canceladas y ese día de muestreo fue repetido posteriormente.

Los datos de comportamiento alimentario fueron tomados con el método de barrido lento ("slow scan", Altmann 1974 adaptado por Robinson 1986; Defler y Defler 1996), cada 15 minutos, 7 de los cuales fueron empleados en la búsqueda del mayor número de individuos posibles. Los barridos inicialmente se planearon hasta de 10 minutos, pero el tamaño pequeño del grupo permitió encontrar a la gran mayoría de integrantes en menor tiempo (7 minutos). Durante los barridos, en caso de consumo, se registró el primer ítem consumido por cada individuo visible (un solo registro por individuo, por barrido). Cada registro fue tomado como un evento de consumo de cada ítem. Se especificó el ítem consumido: frutos (consumo de frutos en cualquier estado de maduración, incluyendo nueces y semillas), partes vegetales (tallos, pecíolos, hojas, médula, raíces), artrópodos (ingestión en cualquier estado de desarrollo: larvas, pupas, adultos), varios (ingestión de otros ítems como por ejemplo tierra de termiteros, miel o cera de panales, agua y pequeños vertebrados). El porcentaje de consumo de los diferentes ítems alimentarios fue expresado a partir del número de registros individuales de consumo para cada tipo de alimento. Para comparar el patrón de alimentación entre los períodos de abundancia, fue realizada una ANOVA de 2 vías, donde los tratamientos fueron los ítems alimentarios y los períodos de oferta de alimento; y las repeticiones cada uno de los meses de estudio (Robinson 1986). Dichos porcentajes fueron normalizados por transformación arcoseno. Para analizar si tanto el consumo mensual de frutos maduros como el consumo mensual de artrópodos variaron de acuerdo a su oferta, se empleó un rango de correlación de Spearman en cada caso. Para describir la forma en que los maiceros manipulaban sus alimentos, fueron empleadas observaciones "ad libitum" (Altmann 1974).

Diversidad mensual de frutos consumidos: Mensualmente se registraron todas las especies de frutos consumidas por los 
maiceros y su consumo. El consumo se obtuvo a partir de los datos tomados durante los barridos lentos. Se calculó la diversidad mensual de frutos consumidos, con el índice de Shannon - Wiener, $\mathrm{H}^{\prime}=-$ - pilogpi, donde pi es el número de registros de consumo de cada especie de fruto, dividida entre el total de registros de consumo de frutos en cada mes (Ludwig y Reynolds 1988).

Selectividad por especie de fruto consumido: Con los datos sobre consumo de cada especie de fruto y su disponibilidad en los transectos de fenología, se calculó el índice de selectividad de Jacob (Stevenson et al. 2000): Jacob D = ri-pi / (ri+pi 2 piri). Donde ri $=$ proporción de una especie de fruto en la dieta, $\mathrm{pi}=$ proporción de la especie de fruto en el bosque. $\mathrm{El}$ índice varía entre -1 (fuerte rechazo) y 1 (fuerte preferencia).

Dispersión espacial de árboles con fruto maduro: Cada mes fueron trazados al azar tres transectos de $500 \times 20 \mathrm{~m}$, dentro de éstos se estableció una cuadrícula de 10 x $10 \mathrm{~m}$. En cada cuadrado se contó el número de árboles con fruto de aquellas especies consumidas por los maiceros (registradas durante los barridos lentos). Con estos datos se determinó la dispersión de los recursos utilizados por los maiceros en cada mes, empleando el índice de varianza / media, ID $=s^{2} / x$ (Ludwig y Reynolds 1988). Si la muestra sigue una distribución de Poisson, el ID es 1. La diferencia de 1 se puede probar por medio de una prueba de $X^{2}$ y el test estadístico $d\left(\sqrt{2} X^{2}-\sqrt{ }(2-\mathrm{N})-1\right)$.

Densidad de árboles con fruto maduro: Cada uno de los árboles visitados por el grupo de maiceros para alimentarse de fruto cada mes, fue localizado en el mapa del área de estudio. La densidad fue calculada dividiendo el número de árboles por el tamaño del área de actividad del grupo de maiceros (Spironello 2001).

Tasa de éxito de captura de artrópodos: Después de los barridos lentos, cuando el grupo se encontraba forrajeando artrópodos, fue estimada la tasa de éxito de captura. Esta tasa fue tomada como el número de artrópodos capturados por un maicero durante cinco minutos continuos de observación, empleando el método "animal focal" (Altmann 1974). Cada intento exitoso por parte del animal focal para capturar un artrópodo fue registrado como un evento de captura. Se definió intento exitoso como el salto, movimiento o manipulación realizada por el animal focal hacia un artrópodo, que finalizó con el consumo del mismo (Melin et al. 2007). Las observaciones se alternaron entre los individuos del grupo, para obtener similares tiempos de observación entre sexos y edades. Se empleó una prueba de Kruskal-Wallis para comparar las tasas de captura entre temporadas de oferta de recursos. Para analizar si los cambios en la oferta de artrópodos, resultaron en un cambio en el éxito de captura, se correlacionaron dichas variables a lo largo del ańo (Spearman $\mathrm{r}_{\mathrm{s}}$ ).

La normalidad de todos los datos del estudio fue probada con test de Shapiro - Wilk.

\section{RESULTADOS}

\section{Oferta de recursos}

Artrópodos: El número de artrópodos colectados varió a lo largo del estudio ( $\mathrm{F}=2.33 \mathrm{gl} .=11,120 \mathrm{p}<0.01)$ (Figura 2a). Fueron colectados 16998 individuos con una media mensual de $1416.5 \pm 274.8$. Éstos pertenecieron a 23 grupos: 17 órdenes y 6 categorías, que incluyeron otros estadíos y familias. El grupo más abundante fue Formicidae con el $47.4 \%$ de los individuos colectados. Coleoptera y Arachnida ocuparon aproximadamente el $11 \%$ cada uno, y los huevos y larvas de diferentes órdenes, que son alimento de insectívoros, $12 \%$ (Anexo 1). Los grupos colectados fueron más numerosos durante el período de abundancia que en el de escasez ( $\mathrm{t}$ pareada $=2.05 \mathrm{gl} .=22 \mathrm{p}=0.05$ ), a excepción de Thysanoptera y Psocoptera. Así, la abundancia de artrópodos en la EBMIC no fue constante a lo largo del año, presentando períodos de baja disponibilidad para los maiceros.

Frutos maduros: El número de árboles con frutos maduros se correlacionó con el número total de frutos maduros contados por transecto, cada mes $\left(\mathrm{r}_{\mathrm{s}}=0.81 \mathrm{n}=12 \mathrm{p}<0.01\right)$. Por tanto,
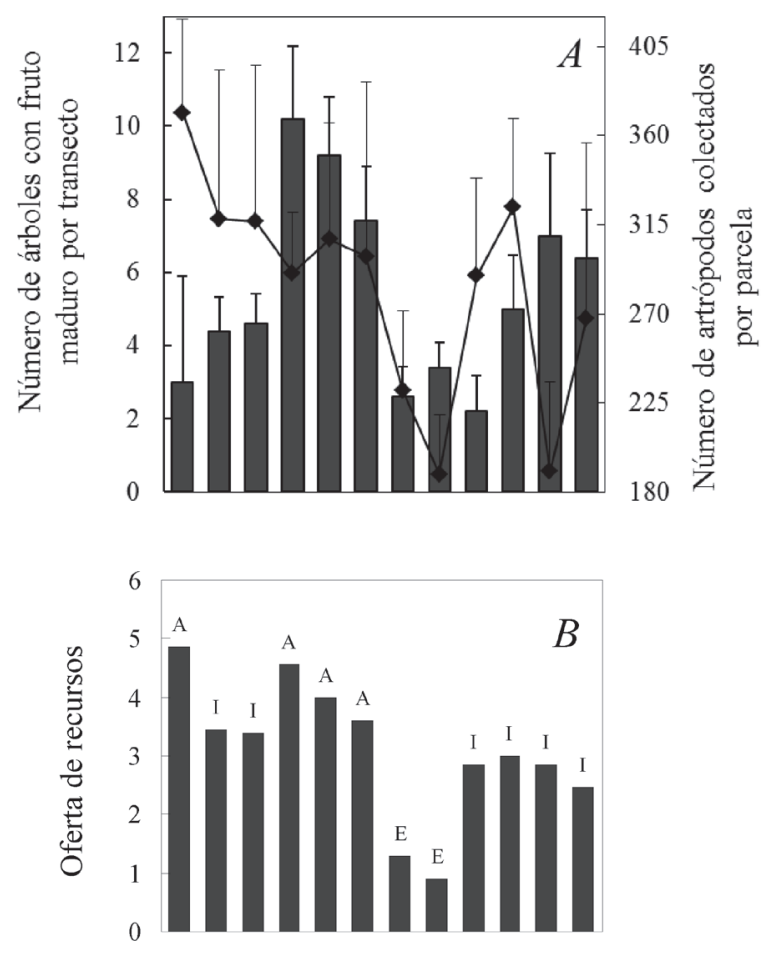

E

Meses

Figura 2 - Oferta mensual de recursos durante 1999. 2A. Oferta de artrópodos y árboles con fruto maduro (promedio mensual y error estándar). Las barras indican el número de árboles y la línea el número de artrópodos. 2B. Oferta combinada de estos dos recursos: $A=$ abundante, $I=$ intermedia, $E=$ =escasez. 
se trabajó con el número de árboles como indicativo de la disponibilidad de fruto maduro. A lo largo del año, el número de árboles con frutos varió $(\mathrm{H}=26.9 \mathrm{gl} .=11,60 \mathrm{p}<0.01)$ (Figura 2a). Los picos de abundancia se encontraron de abril a junio. Durante enero, las palmas de cumare (Astrocaryum aculeatum) maduraron sincrónicamente, $\mathrm{y}$ fueron un recurso importante y muy abundante para $C$. apella. En febrero, los principales recursos fueron diferentes especies de guamas (Inga spp.). En abril, mayo y junio, época de amplia oferta, gran variedad de árboles fructificaron de forma abundante, como por ejemplo Doliocarpus cf. dentatus, Clusia sp., Strychnos cf. erichsonii y Philodendron sp. En julio y principalmente en agosto, meses de escasez de frutos, la coca Cariniana micrantha (Lecythidaceae) fue una de las pocas especies que ofreció frutos. En octubre, período seco, varias especies de mamitos (Virola spp. e Iryanthera spp. - Myristicaceae) fueron abundantes. En noviembre y diciembre, los uvos de monte (Pourouma spp.) fructificaron en grandes proporciones.

Oferta combinada: los meses en que coincidió la abundancia de frutos y de artrópodos fueron: enero, abril, mayo y junio. Los de escasez fueron julio y agosto. Los meses restantes presentaron una oferta intermedia (Figura $2 b$ ). Aunque en enero se encontraron pocos árboles con fruto maduro en el transecto de fenología, se incluyó este mes como abundante debido a la gran oferta de $A$. aculeatum, de distribución agrupada y reconocida importancia en la dieta de los maiceros (Izawa 1979; Peres 1991; Terborgh 1983).

\section{Dieta y comportamiento alimentario}

La dieta de los maiceros fue principalmente insectívora - frugívora. De los 6354 registros de alimentación, 48.8\% fueron en artrópodos y $42.9 \%$ en fruto. Las partes vegetales se utilizaron en un $6.7 \%$ y varios $1.6 \%$.

El grupo en estudio utilizó 96 especies de plantas pertenecientes a 35 familias. Las familias con mayor cantidad de géneros consumidos fueron Arecaceae, seguida por Moraceae y Myristicaceae; y las de mayor porcentaje de consumo Arecaceae (18.1\%) y Lecythidaceae (13.3\%) (Anexo 2). El fruto más consumido fue $A$. aculeatum (cumare, $13.8 \%$ ), seguido de C. micrantha (coca, 10.6\%), Strychnos cf. erichsonii (8.5\%), Inga spp. (guamas, 6.2\%), Pourouma spp. (uvos de monte, $5.9 \%$ ), Philodendron sp. (5.3\%), Virola spp. (mamitos $2.1 \%$ ) e Iryanthera spp. (mamitos $1.3 \%$ ). La mayoría de los frutos consumidos fueron carnosos, a excepción de los frutos duros de A. aculeatum, C. micrantha y Sterculia cf. apetala. Estos frutos implicaron destreza, fuerza y tiempo para abrirlos (deben golpearlos contra troncos de árboles). Los maiceros consumieron frutos inmaduros de las palmas A. aculeatum, Iriartea ventricosa (bombona), Euterpe catinga (asaí), Oenocarpus bataua (milpeso) y Oenocarpus mapora (milpesillo). Dentro de su dieta, los maiceros depredaron las semillas de 5 especies: $C$. micrantha, Micrandra spruceana (yetcha), Cuoroupita guianense (castaño), Sandwithia heterocalyx y Gustavia hexapetala.

Las partes vegetales más consumidas fueron plántulas, tallos y médula de palmas jóvenes, hojas nuevas de $A$. aculeatum, E. catinga, I. ventricosa, Socratea exorrhiza (zancona), Bromeliaceae, Araceae (Monstera adansonii, Philodrendron sp.) y Cyclanthaceae, zona apical de bejucos y raíces de diferentes epífitas (anexo 2). El consumo de partes vegetales fue generalmente oportunista y esporádico y excepto las hojas, obtenerlas y manipularlas implicó fuerza y destreza.

Los maiceros buscaron artrópodos principalmente en sustratos específicos como por ejemplo en hojas enrolladas, suspendidas o acumuladas en ramas, dentro de madera podrida, debajo de la corteza de árboles y copa de palmas. No se pudo cuantificar el porcentaje de consumo de los diferentes grupos capturados; sin embargo, se identificó el consumo de hormigas (Formicidae): adultos, larvas, pupas y huevos; termitas (Isoptera): adultos, ninfas y huevos; arañas (Arachnidae): adultos y posiblemente huevos; saltamontes (Orthoptera, principalmente Tettigonidae): adultos; escarabajos (Coleoptera): larvas y pupas; y larvas y huevos de diferentes grupos como hymenoptera (principalmente avispas), hemiptera y lepidoptera. Se observó el consumo de larvas del escarabajo Rynchophorus cf. palmarum (mojojoi, Colepotera: Curculionidae). Estas larvas se encuentran dentro de troncos de palmas y los maiceros las atraparon rompiendo la corteza, o usando astillas de madera. El consumo de estas larvas siempre causó gran actividad y excitación en el grupo.

En pocas ocasiones (varios $=1.6 \%$ de la dieta) se les observó consumiendo otros ítems como tierra de termiteros, miel de abejas, pequeños roedores (Oecomys sp.), ardillas (Sciurus igniventris), ranas, huevos de rana, lagartijas, huevos y polluelos de aves. Para el consumo de mamíferos y ranas, los maiceros siempre empezaron a consumir los dedos (mientras la presa estaba aun viva), siguiendo con las extremidades, cabeza y por último el tronco. La presa siempre fue sujetada por el tronco, lo cual produjo su muerte por asfixia. Para el caso de las ranas de piel con muchas glándulas, los maiceros retiraron la piel, restregando el cuerpo de la rana contra la corteza de los árboles. Los maiceros generalmente consumieron el 100\% de la presa, dificultando su identificación.

Variación mensual en la dieta: La proporción de los diferentes ítems en la dieta de los maiceros varió dependiendo de la temporada de oferta de recursos $(\mathrm{F}=5.15 \mathrm{gl} .=6,32$ $\mathrm{p}<0.01$ ) (Tabla 1). Siempre fueron los frutos y los artrópodos los más consumidos. Las partes vegetales y otros ítems (categoría varios) fueron utilizados en menor proporción. Durante los meses de abundancia de recursos, fueron preferidos los frutos; su consumo disminuyó hasta un 35\% en el período de escasez. En este último, el ítem principal fueron los artrópodos; sin embargo, su consumo fue menor al 50\% 
de la dieta. El mayor consumo de artrópodos se presentó en los meses de disponibilidad intermedia y no en los de escasez.

El consumo mensual de frutos se incrementó a medida que los maiceros encontraron mayor disponibilidad de frutos maduros en el bosque $\left(r_{s}=0.71 n=11 p=0.01\right)$. Cuando la oferta de frutos decayó, los maiceros complementaron la dieta con artrópodos. Así, el porcentaje mensual de artrópodos en la dieta se correlacionó de forma negativa con el porcentaje del consumo de frutos $\left(r_{s}=-0.69 n=11 p=0.02\right)$ y con la oferta de éstos en el bosque $\left(r_{\mathrm{s}}=-0.61 \mathrm{n}=11 \mathrm{p}=0.04\right)$. Cuándo frutos $\mathrm{y}$ artrópodos escasearon, la dieta fue complementada con partes vegetales y otros ítems (varios); cuyos porcentajes de consumo se incrementaron alrededor del doble durante el período de escasez (Tabla 1).

En dicho período de escasez de recursos (julio y agosto), no solo el porcentaje de consumo de partes vegetales fue incrementado (Tabla 1), también el número de especies de las cuales consumieron partes vegetales fue aproximadamente el doble (Tabla 2). En estos meses, dentro de los principales ítems y especies consumidas, se encontraron pecíolos de Cyclanthaceae sp., hojas nuevas de $M$. adansonii y hojas nuevas de I. ventricosa. La coca (C. micrantha) fue básicamente

Tabla 1 - Porcentaje de consumo de ítems alimentarios por $C$. apella de acuerdo a la oferta combinada de frutos y artrópodos en la EBMIC

\begin{tabular}{cccccc}
\hline Oferta recursos & \% Artrópodos & \% Frutos & \% P. vegetales & \% Varios & $\mathrm{N}$ \\
\hline Abundancia & 44.0 & 51.1 & 4.9 & 0 & 2678 \\
Intermedia & 55.1 & 37.5 & 5.3 & 2.2 & 2562 \\
Escasez & 45.7 & 35.8 & 14.2 & 4.3 & 1114 \\
\hline Total 11 meses & 48.8 & 42.9 & 6.7 & 1.6 & 6354 \\
\hline
\end{tabular}

Tabla 2 - Número de especies de plantas consumidas, diversidad, dispersión y densidad mensual de árboles con fruto maduro consumidos por los maiceros, y tasa de éxito de captura de artrópodos. $\mathrm{G}=$ dispersión agrupada. $\mathrm{R}=$ dispersión al azar

\begin{tabular}{|c|c|c|c|c|c|c|c|}
\hline \multirow[t]{2}{*}{ Mes } & \multicolumn{2}{|c|}{$\begin{array}{l}\text { Número especies } \\
\text { consumidas }\end{array}$} & \multirow{2}{*}{$\begin{array}{c}\text { Diversidad } \\
\mathrm{H}^{\prime}\end{array}$} & \multirow{2}{*}{\multicolumn{2}{|c|}{$\begin{array}{c}\text { Dispersión } \\
\text { ID }\left(s^{2} / x\right) \\
{ }^{*} p<0.05\end{array}$}} & \multirow{2}{*}{$\begin{array}{c}\text { Densidad } \\
\text { Árboles/ } \\
\text { ha }\end{array}$} & \multirow{2}{*}{ 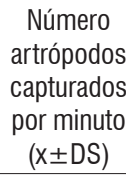 } \\
\hline & Frutos & P. vegetales & & & & & \\
\hline Ene & 15 & 4 & 1.47 & $3.33^{*}$ & $\mathrm{G}$ & 0.65 & $4.2 \pm 0.6$ \\
\hline Feb & 14 & 0 & 2.03 & 0.86 & $\mathrm{R}$ & 0.40 & $2.8 \pm 0.4$ \\
\hline$A b r$ & 19 & 2 & 2.31 & $1.53^{\star}$ & $\mathrm{G}$ & 0.36 & $2.5 \pm 0.7$ \\
\hline May & 20 & 4 & 1.82 & $2.10 *$ & $G$ & 0.63 & $2.4 \pm 0.6$ \\
\hline Jun & 25 & 5 & 2.57 & $1.97^{\star}$ & G & 0.38 & $3.2 \pm 0.2$ \\
\hline Jul & 8 & 9 & 1.82 & 1.03 & $\mathrm{R}$ & 0.10 & $1.8 \pm 0.9$ \\
\hline Ago & 9 & 7 & 1.07 & 0.82 & $\mathrm{R}$ & 0.17 & $1.9 \pm 0.1$ \\
\hline Sept & 9 & 2 & 1.70 & 0.91 & $\mathrm{R}$ & 0.05 & $2.4 \pm 0.3$ \\
\hline Oct & 12 & 4 & 2.94 & 1.03 & $\mathrm{R}$ & 0.20 & $2.2 \pm 1.0$ \\
\hline Nov & 9 & 2 & 1.45 & $1.28 *$ & $G$ & 0.23 & $1.8 \pm 0.6$ \\
\hline Dic & 15 & 3 & 1.94 & $2.96^{\star}$ & $\mathrm{G}$ & 0.17 & $2.0 \pm 0.4$ \\
\hline
\end{tabular}

el único fruto disponible en estos meses de baja oferta. Sus semillas fueron explotadas intensivamente por los maiceros $(51.6 \pm 21.9 \%$ de los registros de consumo de frutos en dichos meses). Adicionalmente, en julio, una palma de cumare ( $A$. aculeatum) fructificó asincrónicamente. El grupo la visitó en repetidas ocasiones. Gracias a estas dos especies, el consumo de frutos no cayó tan drásticamente durante la escasez.

Diversidad mensual de frutos consumidos: Se esperaba que una disminución en la oferta de frutos, estuviera relacionada con una dieta menos selectiva en la escogencia de alimentos y por tanto con una mayor diversidad. Sin embargo, no se encontró una relación entre la disponibilidad mensual de árboles con fruto y diversidad de los frutos consumidos $\left(r_{\mathrm{s}}=0.45 \mathrm{gl} .=11 \mathrm{p}=0.1\right)$ (Tabla 2). Por ejemplo, en agosto hubo muy poca oferta de fruto y los maiceros usaron intensivamente la coca C. micrantha ( $72 \%$ de los registros de consumo de frutos en este mes); mostrando una diversidad baja (Tabla 2). En mayo, se presentó una alta oferta de diferentes frutos, sin embargo, los maiceros consumieron intensamente Strychnos cf. erichsonii (51\% de los registros de consumo de frutos), presentando un valor bajo de diversidad (Tabla 2).

Selectividad por especie de fruto consumido: Los datos arrojaron una media de $-0.22 \pm 0.37$ que denotan una muy baja selectividad en la dieta. Los maiceros mostraron una dieta generalista - oportunista, con una no muy alta preferencia por unas pocas especies como $S$. cf. apetala (0.54) y $C$. micrantha (0.44).

Dispersión espacial de árboles con fruto maduro: La distribución espacial de los árboles con fruto maduro usados por los maiceros fue variable a lo largo del estudio y no se encontró una relación entre la dispersión y la disponibilidad mensual de fruto $\left(r_{s}=0.42 n=11 \mathrm{p}=0.18\right)$. Sin embargo, los meses con mayor oferta de fruto presentaron una distribución agrupada (Tabla 2). En los demás meses, la distribución de los árboles con frutos consumidos por los maiceros fue al azar.

Densidad mensual de árboles con fruto maduro: El grupo visitó 481 árboles de alimentación durante todo el período de estudio, lo cual arroja una densidad de 1.5 árboles/ha. En los meses de mayor oferta de frutos se observó una leve tendencia del grupo a usar una mayor densidad de árboles con fruto maduro $\left(r_{s}=0.51 \mathrm{n}=11 \mathrm{p}=0.07\right)$ (Tabla 2).

Tasa de éxito de captura de artrópodos: Durante el forrajeo de artrópodos, los maiceros tuvieron una tasa de captura de $2.5 \pm 3$ individuos por minuto (Tabla 2). Los episodios de búsqueda continua de artrópodos no fueron largos (51.7 29.9 segundos, con un máximo de 303 segundos). Las tasas de éxito de captura variaron entre temporadas de oferta de recurso $(\mathrm{H}=7.2 \mathrm{gl} .=2,37 \mathrm{p}=0.03)$. En los meses de escasez de artrópodos, las tasas de captura fueron de $1.8 \pm 0.1$, en la temporada de oferta intermedia de $2.2 \pm 0.4$ y en abundancia de $3.1 \pm 0.8 \mathrm{ind} /$ minuto. La oferta mensual de artrópodos se 
correlacionó con la tasa de captura $(\mathrm{rs}=0.76 \mathrm{n}=11 \mathrm{p}=0.01)$. Es decir, los maiceros atraparon mayor cantidad de artrópodos cuando la disponibilidad de éstos fue mayor.

\section{DISCUSIÓN}

\section{Oferta de recursos}

Abundancia de artrópodos: Las fluctuaciones numéricas en la abundancia de artrópodos pueden causar estrés por escasez, en los vertebrados que se alimentan de ellos (Boinski y Fowler 1989) e incluso pueden ocasionar cambios en las tácticas de los depredadores (Terborgh 1983; Poulin et al. 1992; Rímoli et al. 2008). La mayor abundancia de artrópodos en la EBMIC correspondió, en general, con el período de lluvias; disminuyendo durante los meses de menor precipitación. En las selvas de tierras bajas se ha demostrado que la estacionalidad de la precipitación causa una respuesta numérica en las poblaciones de artrópodos. La lluvia puede determinar su abundancia a través de efectos directos en la fisiología (como por ejemplo aumento en la ovoposición, reproducción, desarrollo y actividad) e indirectamente puede afectar el crecimiento de las plantas y por consiguiente, la disponibilidad de alimento para artrópodos (Janzen 1973; Boinski y Fowler 1989).

Los maiceros son primates principalmente de sotobosque, que utilizan el bosque generalmente a una altura alrededor de los 6 ó $7 \mathrm{~m}$. Sin embargo, es común verlos a alturas menores y en el suelo, así como en el dosel (Terborgh 1983; Janson y Boinski 1992). El muestro manual realizado en este estudio esta limitado solamente hasta los $3 \mathrm{~m}$ de altura, lo que sesga los resultados a insectos de sotobosque. Sin embargo, Barone (2000) encontró que los picos de abundancia de insectos durante la producción de hojas nuevas son similares en el dosel y en el sotobosque, tanto en la densidad de individuos, como en la riqueza de los grupos que se ven favorecidos en estos períodos. Es decir, que los resultados del muestreo en la EBMIC, aunque sesgados al sotobosque, pueden reflejar los patrones de abundancia de artrópodos en el bosque.

Cebus apella y C. capucinus generalmente no capturan artrópodos al vuelo y no los buscan al azar. Estas especies escudriñan en sustratos específicos, donde la señal como tal no es la presa, si no el sustrato mismo (Janson y Boinski 1992; Melin et al. 2007). Los artrópodos capturados por los maiceros, en general, no tratan de escapar volando, sino que se esconden dentro de sustratos específicos (Fragaszy et al. 2004). En este trabajo, los órdenes que más se colectaron fueron los consumidos por los maiceros. Y se encontró una concordancia entre tasas de captura y abundancia de artrópodos. Es decir, que un muestreo manual puede ser eficaz para colectar el tipo de presas que consumen los maiceros (Boinski y Fowler 1989; Melin et al. 2007) y parece ser un buen indicador de la disponibilidad de presas para estos primates.
Oferta de frutos: El patrón fenológico encontrado para la producción de frutos en EBMIC concuerda con el obtenido por Defler y Defler (1996) durante 4.5 ańos de seguimiento a algunos árboles de importancia para primates en la misma zona. Los patrones fenológicos en los bosques tropicales indican una estacionalidad en la disponibilidad de las partes reproductivas de las plantas, resultando en un cuello de botella para muchas especies frugívoras. Estos deben migrar o buscar alimentos alternativos, generalmente de menor recompensa energética o de difícil obtención (Peres 1994).

Los meses de mayor oferta de fruto maduro para los maiceros en la EBMIC presentaron una amplia abundancia de este recurso. En estos meses de amplia oferta, los árboles con fruto maduro tuvieron una distribución agrupada, relacionado con una alta densidad de los mismos en el área de actividad de los maiceros. Por el contrario, en los meses de escasez, hubo muy poca oferta de fruto maduro. Los suelos de la EBMIC son pobres en nutrientes y de baja fertilidad (Defler y Defler 1996). Esta situación resulta en cosechas de frutos de menor calidad y cantidad que en otras zonas de bosques de tierras bajas (Gentry y Emmons 1987). Por consiguiente, los períodos de escasez de frutos son muy drásticos para grandes frugívoros en esta región (Defler y Defler 1996).

\section{Dieta y comportamiento alimentario}

La disponibilidad temporal de frutos y artrópodos, la variabilidad de su contenido nutritivo y la necesidad de alternar alimentos, favorecen una dieta flexible para primates tropicales como C. apella. La adaptabilidad de los maiceros a una gran diversidad de hábitats, en los cuales despliegan una amplia variación ecológica y conductual, ha sido reportada por diversos autores (eg. Brown y Zunino 1990; Zhang 1995; Freitas et al. 2008). La dieta omnívora de los maiceros en la EBMIC corresponde con lo reportado para la especie, siendo principalmente insectívoros-frugívoros y usando una amplia variedad de recursos, que incluyeron frutos duros de difícil manipulación, partes vegetales y vertebrados (Fragaszy et al. 2004).

Los cambios estaciónales en la dieta de los maiceros en la EBMIC fueron en general congruentes con la oferta de recursos. Durante los períodos de amplia oferta, la dieta incluyó más de 20 especies de frutos por mes y las tasas de captura de artrópodos fueron mayores. Por el contrario, durante los períodos de escasez, la dieta incluyó menos de 10 especies de frutos por mes y las tasas de captura de artrópodos fueron bajas. El fruto disponible y más importante para los maiceros en este período, fue la coca, $C$. micrantha (Lecythidaceae). Este fruto duro y resistente implicó fuerza y experiencia para su manipulación. En estos meses, los maiceros incrementaron el consumo de plántulas y médula de diferentes palmas y hojas nuevas de bromelias, ciclantáceas y aráceas, las cuales debieron arrancar y manipular con destreza. La fuerte 
musculatura de las mandíbulas y brazos de los maiceros les permite utilizar una amplia variedad de recursos, muchas veces inaccesibles para otras especies de primates (Fragaszy et al.2004). Este tipo de recursos son utilizados principalmente durante las temporadas de escasez de frutos carnosos. Así por ejemplo Terborgh (1983) y Spironello (1991) reportan durante las temporadas de escasez el consumo de nueces como crítico para los maiceros. En Brasil, durante la escasez de frutos carnosos los maiceros consumieron semillas de frutos duros como las cocas Cariniana legalis (Galetti y Pedroni 1994) y C. micrantha (única especie disponible en dichos meses) y complementaron su dieta con néctar y hojas jóvenes (Peres 1991, 1994). Similar estrategia se observó para maiceros en la EBMIC. Durante la temporada de escasez de recursos, éstos incrementaron el consumo de partes vegetales y explotaron intensivamente las semillas de la coca.

En varios estudios se ha reportado que la dieta de los maiceros en los períodos de escasez consta principalmente de artrópodos, siendo complementada con partes vegetales. Ambos ítems presentan los mayores porcentajes de consumo en estas temporadas (eg. Soini 1986; Stevenson et al. 2000; Rímoli et al. 2008). En la EBMIC, la dieta también fue complementada con partes vegetales durante la temporada de escasez de recursos; pero el consumo de artrópodos no superó el 50\% de los ítems utilizados. Durante estos meses los artrópodos también escasearon y las tasas de captura fueron menores. En Manu, Perú, Terborgh (1983) encontró períodos con menor éxito de captura de artrópodos para $C$. apella. Sus resultados sugieren que la estacionalidad en la disponibilidad de artrópodos puede causar una fuerte disminución en el número de individuos capturados; tal como fue encontrado para los maiceros en la EBMIC.

En este estudio, los maiceros no presentaron fuerte selectividad por una especie de fruto en particular. Estos resultados sugieren que en general, los micos utilizaron la oferta de frutos del bosque en la misma proporción en que estuvo disponible. Igual situación se encontró para $C$. apella en Perú (Terborgh 1983) y en Colombia (Stevenson et al. 2000). Allí, la dieta fue generalista, con bajos índices de selectividad.

En ambientes altamente estacionales, la fenología de frutos determina variaciones significativas en la dieta de Cebus spp. y estos primates pueden presentar respuestas contrastantes. Así por ejemplo, durante los períodos de escasez de frutos, estos monos generalmente se concentran en unas pocas especies de frutos claves e incrementan el consumo de partes vegetales. Sin embargo, en estos períodos, el consumo de artrópodos varía ampliamente en diferentes estudios, desde muy poco consumo hasta ser el principal recurso en la dieta: menos del 20 \% hasta 75\% (eg. Peres 1994; Gómez-Posada 2003a; Freitas et al. 2008). Estas diferentes estrategias reportadas en dichos estudios, pueden estar influenciadas por la abundancia de artrópodos durante los períodos de escasez de fruto; dicha abundancia de artrópodos no fue evaluada en ninguno de los estudios.

Podría esperarse que en los lugares con una dieta pobre en especies de frutos, los maiceros presentaran altos niveles de insectivoría. Sin embargo, los resultados de este trabajo demuestran que no solo la disponibilidad de frutos determina la dieta de un animal, también la disponibilidad de artrópodos. Freitas et al. (2008) reportan para un grupo de maiceros el uso de 54 especies de plantas, con un consumo de frutos del $47 \%$ y de material animal solamente del 9\%. Terborgh (1983) reporta 100 especies de plantas en la dieta de un grupo de maiceros, con un bajo consumo de material vegetal ( $16 \%$ de la dieta) y un alto consumo de insectos (50\%). Stevenson et al. (2000) reportan 126 especies de plantas usadas por un grupo de maiceros, con un consumo de frutos del $33 \%$ y de insectos del $43 \%$. La plasticidad de estos primates les permite usar una gran diversidad de recursos alternativos y tener una dieta muy variada, acorde con los recursos de cada localidad. Un animal escoge su alimento con base principalmente en dos factores: valor nutritivo y disponibilidad. Los frutos aportan carbohidratos (energía) y los artrópodos son el principal recurso de proteína para primates como los Cebus. La morfología, fisiología y capacidad cognitiva de los maiceros les permite explotar una amplia gama de recursos, desplegando una patrón de forrajeo altamente adaptativo a cada tipo de hábitat donde se encuentran (Fragaszy et al. 2004).

La habilidad del género Cebus para utilizar alimentos alternativos durante los cuellos de botella de sus dos recursos principales: frutos y artrópodos, es esencial para su éxito en una amplia variedad de bosques neotropicales (Fragaszy et al. 2004) y hace de este género de primates un buen modelo para entender como los hábitats pueden modular las respuestas ecológicas y comportamentales de los primates (Galetti y Pedroni 1994).

\section{AGRADECIMIENTOS}

Este trabajo fue financiado por Fundación Natura/ COLCIENCIAS. A Thomas Defler, director de este proyecto, por la oportunidad de trabajar en Caparú, por su constante apoyo, enseñanzas y amistad, y por la incansable dedicación al estudio de los primates Colombianos. A Michael Alberico $(\dagger)$ codirector de este trabajo, por su gran apoyo, su inigualable humor negro y por compartirme su pasión por los mamíferos. A Conservation International Colombia, por el apoyo y colaboración prestado, especialmente a Erwin Palacios y Adriana Rodríguez. A Marcelino Días y familia, Jaime Castillo y Ángela Maldonado, por su apoyo en campo. A Isidoro Cabrera y Rodrigo Botina por la identificación del material vegetal. 


\section{REFERENCIAS}

Altmann, J. 1974. Observational study of behavior: sampling methods. Behavior, 49: 227-265.

Barone, J. 2000. Comparison of herbivores and herbivory in the canopy and understory for two tropical tree species. Biotropica, 32: 307-317.

Boinski, S. 1987. Habitat use by squirrel monkeys (Saimiri oerstedii) in Costa Rica. Folia Primatologica, 49: 151-167.

Boinski, S.; Fowler, N. 1989. Seasonal patterns in a tropical lowland forest. Biotropica, 21: 223-233.

Brown, A.; Zunino, G. 1990. Dietary variability in Cebus apella in extreme habitats: evidence for adaptability. Folia Primatologica, 54: 187-195.

Defler, T. 2004. Primates of Colombia. Conservation International, Bogotá, Colombia. 550 pp.

Defler, T.; Defler, S. 1996. Diet of a group of Lagothrix lagothricha in southeastern Colombia. International Journal of Primatology, 17: 161-190.

Fragaszy, D.; Visalberghi, E.; Fedigan, L. 2004. The complete capuchin, the biology of the genus Cebus. Cambridge University Press, Cambridge, UK. 356 pp.

Freitas, C.A.; Setz, E.; Araújo, A.; Gobbi, N. 2008. Agricultural crops in the diet of bearded capuchin monkeys, Cebus libidinosus Spix (Primates: Cebidae), in forest fragments in southeast Brazil. Revista Brasileira de Zoologia, 25: 32-39.

Galetti, M.; Pedroni, F. 1994. Seasonal diet of capuchin monkeys (Cebus apella) in a semideciduous forest in south-east Brazil. Journal of Tropical Ecology, 10: 27-39.

Gentry, A.; Emmons, L. 1987. Geographical variation in fertility, phenology, and composition of the understory of neotropical forests. Biotropica, 19: 216-227.

Gómez-Posada, C. 2003a. Foraging strategies of a provisioned group of brown capuchin (Cebus apella) at Tinigua National Park, p. 136-146. In: Pereira, V.; Nassar, F.; Savage, A. (Eds.). New World Primatology. Centro de Primatología Araguatos, Bogotá, Colombia. (in Spanish, with abstract in English).

Gómez-Posada, C. 2003b. Variation in the use of time and space of Cebus apella (Primates: Cebidae) according to the availability of the principals resources in its diet. Master Thesis, Universidad del Valle, Cali, Colombia. 89 pp (in Spansih).

Izawa, K. 1979. Foods and feeding behavior of wild black capped capuchin (Cebus apella). Primates, 20: 57-76.

Janson, C.; Boinski, S. 1992. Morphological and behavioral adaptations for foraging in generalist primates: the case of the Cebinae. American Journal of Anthropology, 88: 483-498.

Janzen, D. 1973. Sweep samples of tropical foliage insects: effects of seasons, vegetation types, elevations, time of day and insularity. Ecology, 54: 687-708.

Ludwig, J.; Reynolds, J. 1988. Statistical Ecology: a primer on methods and computing. John Wiley and Sons, New York, US. 377 pp.
Melin, A.D.; Fedigan, L.M.; Hiramatsu, C.; Sendall C.L.; Kawamura S. 2007. Effects of colour vision phenotype on insect capture by a free- ranging population of white-faced capuchins, Cebus capucinus. Animal Behaviour, 73: 205-214.

Peres, C. 1991. Seed predation of Cariniana micrantha (Lecythidaceae) by brown capuchin monkeys in Central Amazonia. Biotropica, 23: $262-270$.

Peres, C. 1994. Primate responses to phenological changes in an Amazonian terra firme forest. Biotropica, 26: 98-112.

Poulin, B.; Lefebvre, G.; Mcneil, R. 1992. Tropical avian phenology in relation to abundance and explotation of food resources. Ecology, 73: 2295-2309.

Raemaekers, J. 1980. Causes of variation between months in the distances traveled daily by gibbons. Folia Primatologica, 34 : 46-60.

Rímoli, J.; Strier, K.; Ferrari, S. 2008. Seasonal and longitudinal variation in the behavior of free-ranging black tufted capuchins Cebus nigritus (Goldfuss, 1809) in a fragment of Atlantic Forest in Southeastern Brazil, p. 130-146. In: Ferrari, S.; Rímoli, J. (Eds). A Primatologia no Brasil - 9. Sociedade Brasileira de Primatologia, Aracaju.

Robinson, J.G. 1986. Seasonal variation in use of time and space by the wedge-capuchin monkey, Cebus olivaceus: implications for foraging theory. Smithsonian Contributions to Zoology, 431: 1-60.

Rylands, A.B.; Mittermeier, R.A. 2009. The diversity of the New World primates (Platyrrhini): an annotated taxonomy, p. 2354. In: Garber P.A.; Estrada A.; Bicca-Marques J.C.; Heymann E.W.; Strier K.B. (Eds). South American primates: comparative perspectives in the study of behavior, ecology, and conservation. Springer, New York, US.

Soini, P. 1986. A synecological study of a primate community in the Pacaya-Samiria National Reserve, Peru. Primate Conservation, 7: 63-71.

Spironello, W. 1991. Range size of a group of Cebus a. apella in central Amazonia. International Journal of Primatology, 8:522.

Spironello, W. 2001. The brown capuchin monkey (Cebus apella): ecology and home range requirements in central Amazonia, p. 271-283. In: Bierregard, R.O.; Gascon, C.; Lovejoy, T.E.; Mesquita, R. (Eds.). Lessons from Amazonia. Yale University Press, New Haven, US.

Stevenson, P.; Quiñones, M.; Ahumada, J. 2000. Influence of fruit availability on ecological overlap among four neotropical primates at Tinigua National Park, Colombia. Biotropica, 32: 533-544.

Terborgh, J. 1983. Five new world primates. A study in comparative ecology. Princeton University Press, New Jersey, USA. 260 pp.

Zhang, S. 1995. Activity and ranging patterns in relation to utilization by brown capuchins (Cebus apella) in French Guiana. International Journal of Primatology 16: 489-507.

Recebido em: 15/04/2011

Aceito em: 18/10/2011 
ANEXO 1 - Listado de grupos y órdenes de artrópodos colectados por captura manual durante 1999 y porcentaje de abundancia en las capturas (N=16998)

\begin{tabular}{cccccc}
\hline $\begin{array}{c}\text { Grupos y órdenes de } \\
\text { artrópodos }\end{array}$ & $\begin{array}{c}\text { \% total de individuos } \\
\text { colectados }\end{array}$ & $\begin{array}{c}\text { Grupos y órdenes de } \\
\text { artrópodos }\end{array}$ & $\begin{array}{c}\text { \% total de individuos } \\
\text { colectados }\end{array}$ & $\begin{array}{c}\text { Grupos y órdenes de } \\
\text { artrópodos }\end{array}$ & $\begin{array}{c}\% \text { total de } \\
\text { individuos } \\
\text { colectados }\end{array}$ \\
\hline Arachnida & 11.4 & Homoptera & 1.8 & Odonata & 0.1 \\
Blattoidea & 1.0 & Huevos varios & 8.9 & Orthoptera & 3.4 \\
Coleptera & 11.7 & Hymenoptera & 0.5 & Phasmatodea & 0.2 \\
Dermoptera & 1.3 & Isopoda & 0.3 & Pupas varias & 0.1 \\
Diplopoda & 0.2 & Isoptera & 4.9 & Quilopoda & 0.7 \\
Diptera & 2.4 & Larvas varias & 2.9 & Thysanoptera & 0.3 \\
\hline Formicidae & 47.4 & Lepidoptera & 0.2 & & 0.04 \\
\hline Hemiptera & 0.4 & Mantodea & 0.04 & \\
\hline
\end{tabular}

ANEXO 2 - Familias y especies vegetales consumidas por $C$. apella en la EBMIC. En paréntesis está el porcentaje de consumo de cada familia ( $N=2151)$. $\mathrm{IC}=$ ítem consumido, $\mathrm{f}=$ fruto (pulpa), $\mathrm{a}=$ fruto (arilo), $\mathrm{h}=$ hoja nueva, $\mathrm{I}=$ flor, $\mathrm{p}=$ pecíolo, $\mathrm{s}=$ semilla, $\mathrm{t}=$ tallo.

\begin{tabular}{|c|c|c|c|c|c|c|c|}
\hline \multirow{2}{*}{\multicolumn{2}{|c|}{ FAMILIA / Especie }} & & & & \multirow[b]{2}{*}{ C } & & \\
\hline & & FAMILIA / Especie & IC & FAMILIA / Especie & & FAMILIA / Especie & IC \\
\hline ANACARDIACEAE (1.5\%) & & Pourouma cecropiifolia & $f$ & LECYTHIDACEAE (13.3\%) & & Perebea mollis & $f$ \\
\hline Anacardium giganteum & $f$ & Pourouma cucura & $f$ & Cariniana micrantha & $\mathrm{s}$ & Pseudolmedia laevigata & $f$ \\
\hline Spondias mombin & $f$ & Pourouma guianensis & $f$ & Couroupita guianensis & $\mathrm{s}$ & MYRISTICACEAE (3.4\%) & \\
\hline ANNONACEAE (2\%) & & Pourouma minor & $f$ & Eschweilera sp. & $f$ & Iryanthera macrophylla & $\mathrm{a}$ \\
\hline Annona ambotay & $f$ & Pourouma melinonii & $f$ & Gustavia hexapetala & fs & Iryanthera paraensis & $\mathrm{a}$ \\
\hline Annona glabra & $f$ & Pourouma sp. & s & LOGANIACEAE (8.7\%) & & Iryanthera sp. & $a$ \\
\hline Duguettia sp. & $f$ & CELASTRACEAE (0.7\%) & & Strychnos cf. erichsonii & $f$ & Iryanthera ulei & $\mathrm{a}$ \\
\hline Oxandra xylopioides & $f$ & Goupia glabra & $f$ & Strychnos sp. & $f$ & Virola melinonii & $\mathrm{a}$ \\
\hline Xylopia sp. & f & CLUSIACEAE (0.6\%) & & MALPIGHIACEAE $(0.6 \%)$ & & Virola michelii & $\mathrm{a}$ \\
\hline APOCYNACEAE (0.3\%) & & Clusia sp. & f & Byrsonima sp. & $f$ & Virola pavones & $\mathrm{a}$ \\
\hline Couma macrocarpa & f & Garcinia microphylla & f & MELASTOMATACEAE & & Virola sp. & $\mathrm{a}$ \\
\hline ARACEAE (10.3\%) & & CYCLANTHACEAE $(0.2 \%)$ & & & & Virola surinamensis & $\mathrm{a}$ \\
\hline Monstera adansonii & $\mathrm{h}$ & CGP 103 & $p$ & Miconia sp. & $\mathrm{fl}$ & Virola venosa & $\mathrm{a}$ \\
\hline Philodendron sp. & $\mathrm{fh}$ & DILENIACEAE (2.6\%) & & Menispermaceae & & RUBIACEAE (0.6\%) & \\
\hline ARECACEAE (18.1\%) & & Doliocarpus cf. dentatus & $f$ & Caryomene sp. & f & Capirona sp. & $f$ \\
\hline Astrocaryum aculeatum & fhl & EUPHORBIACEAE (0.6\%) & & MONIMIACEA (0.4\%) & & Borojoa amazonica & $f$ \\
\hline Euterpe catinga & fhl & Micrandra spruceana & $\mathrm{s}$ & Siparuna sp. & $f$ & SAPINDACEAE (0.3\%) & \\
\hline Iriartea ventricosa & fhl & Sandwithia heterocalyx & s & OLACACEAE $(0.2 \%)$ & & Paullinia sp. 1 & $f$ \\
\hline Oenocarpus bataua & f & FABACEAE/ & & Minquartia guianensis & f & Paullinia sp. 2 & $f$ \\
\hline Oenocarpus mapora & $f$ & CESALPINOIDE (0.3\%) & & PASSIFLORACEAE (0.5\%) & & SAPOTACEAE (2.3\%) & \\
\hline Scheelea sp. & $f$ & Hymenaea sp. & $f$ & Passiflora sp. & $f$ & Manilkara amazonica & $f$ \\
\hline Socratea exorrhiza & $\mathrm{h}$ & FABACEAE/ & & POLYGALACEAE (0.3\%) & & Manilkara sp. & $f$ \\
\hline BOMBACACEAE (0.3\%) & & MIMOSOIDE (7.1\%) & & Moutabea sp. & $f$ & Pouteria cuspidata & $f$ \\
\hline Castotemma sp. & $f$ & Enterolobium schomburgkii & s & MORACEAE (8.6\%) & & Pouteria sp. & $f$ \\
\hline BROMELIACEAE (0.3) & & Inga alba & f & Brosimum utile & $f$ & Pouteria surinamensis & $f$ \\
\hline CGP 066 & $\mathrm{~h}$ & Inga cinnamomea & $f$ & Castilla ulei & $f$ & STERCULIACEAE (3.5\%) & \\
\hline CGP 154 & $\mathrm{~h}$ & Inga ingoides & $f$ & Ficus guianensis & $f$ & Theobroma subincanum & f \\
\hline BURSERACEAE (1.7\%) & & Inga pezizifera & f & Ficus matthewsii & $f$ & Sterculia cf. apetala & $f$ \\
\hline Protium sp. & $f$ & Inga sp. 1,2,3, and 4 & f & Ficus pertusa & $f$ & STRELITZACEAE (0.8\%) & \\
\hline Protium sagotianum & $f$ & Inga splendens & $f$ & Ficus sp. & $f$ & Phenakospermum & $f$ \\
\hline Tetragastris sp. & f & HIPPOCRATEACEAE (0.3\%) & & Helicostylis tomentosa & $f$ & guyannense & It \\
\hline CARYOCARACEAE (0.2\%) & & Peritassa sp. & f & Naucleopsis calanaeura & $f$ & TILICACEAE (1.1\%) & \\
\hline Caryocar sp. & f & Salacia elliptica & $f$ & Naucleopsis guianensis & $f$ & Apeiba aspera & $f$ \\
\hline CECROPIACEAE (6.1\%) & & LAURACEAE (0.8\%) & & Naucleopsis imitans & $f$ & VITACEAE (0.3\%) & \\
\hline Cecropia obtusa & $\mathrm{fl}$ & Nectandra sp. & $f$ & Naucleopsis amara & $f$ & Cissus sp. & $f$ \\
\hline
\end{tabular}

